\title{
Wave Front Tracking using Template Matching and Segmented Regression
}

\author{
Eirik Siljan Samee Maharjan Marius Lysaker \\ Department of process, energy and environmental technology, University College of Southeast Norway, Norway, \\ 120541@student.hit.no, samee.maharjan@usn.no, marius.lysaker@usn.no
}

\begin{abstract}
An experimental study was designed to measure shock waves (supersonic gas flow) in a shock tube. A highspeed camera captured images of the shock waves, at a framerate up to 500,000 frames per second. With respect to the huge number of images to be analyzed, an imageprocessing algorithm was developed for automatic tracking of the shock waves. However, each shock wave might be divided into to two parts; a normal shock (the shock wave is perpendicular to the flow direction), and an oblique shock (the shock is at an oblique angle relative to the flow direction).

The proposed framework calculates the characteristics of the wave front, i.e. the angle and velocity of normal and oblique shocks. A technique based on Template Matching and an extended version of Segmented Regression is developed to track the wave front in the high-speed videos.

To our understanding, the proposed framework is novel, and our findings are in accordance with results derived from pressure sensors within the test tube
\end{abstract}

Keywords: front tracking, image processing template matching.

\section{Introduction}

This paper introduces a framework for automatic tracking of shock wave fronts in high-speed video films. One major challenge is that high-speed video films are often blurred and corrupted with noise, and accurate physical measurements of key elements within the wave front are difficult to obtain. Figure 1 illustrates wave front propagation throughout a high-speed video.

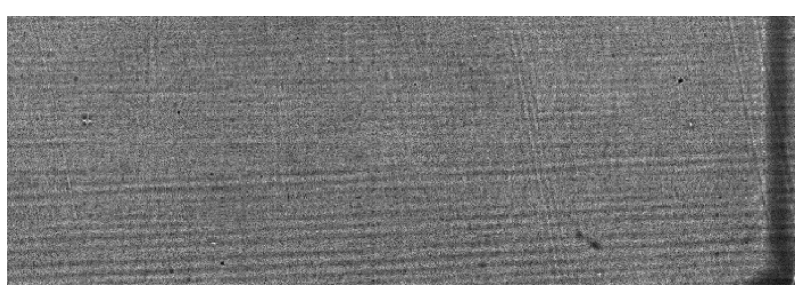

(a)

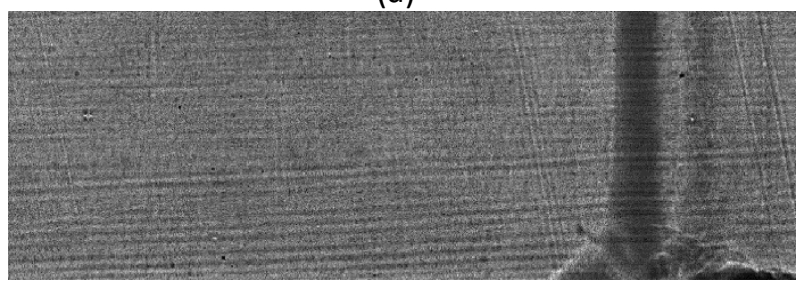

(b)

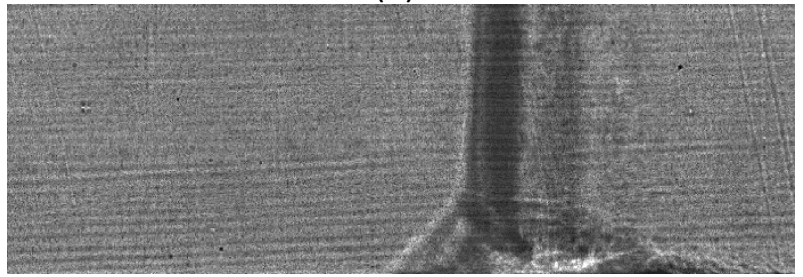

(c)

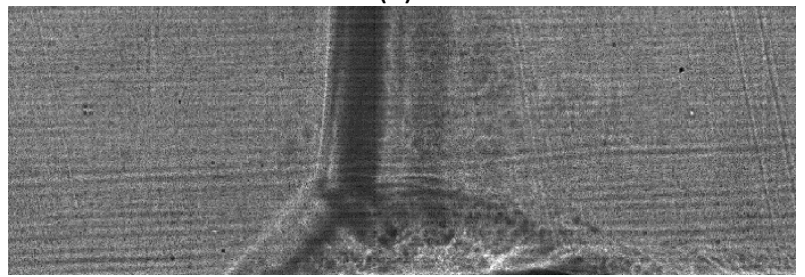

(d)

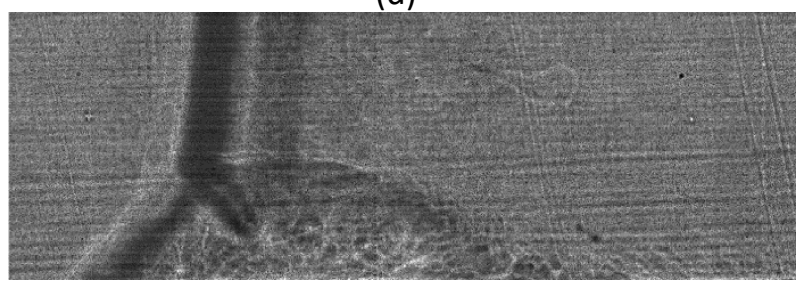

(e)

Figure 1. Illustration of wave front propagation through the test section of the test rig. Images (a) through (e) show frames from 62 through 142 with a 20 -frame interval between each frame. 
Figure 2 illustrates elements of interest within a wave front which are normal and oblique shock angles and velocities.

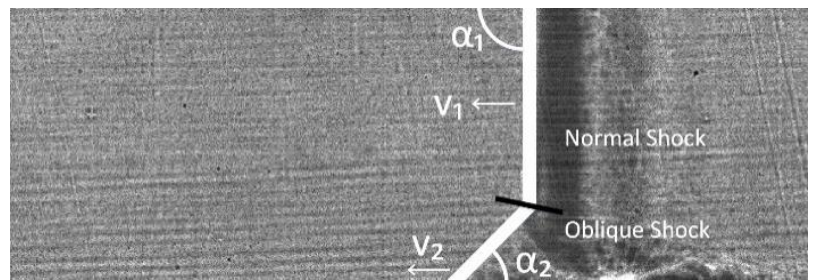

Figure 2. Illustration of key elements within a wave front where $\alpha_{1}$ and $\alpha_{2}$ are normal and oblique shock angles, and $v_{1}$ and $v_{2}$ are normal and oblique shock velocities, respectively.

It is observed in Figure 2 that the normal and oblique shock appears in approximately 90- and 45-degree orientations, relative to the horizontal plane. This orientation of the normal and oblique shocks is also the case throughout multiple videos which are studied.

\section{Methods}

This chapter describes the test rig and methods that first generates two datasets which are later combined using Segmented Regression.

\subsection{Test Rig}

The wave front is generated by a test rig where a flammable gas mixture is pumped into the shock tube, then ignited. The rest of the tube is filled with a specific gas to examine wave front velocities through the selected gases. Among the gases in which the wave front velocity is studied are Ar, N2 and CO2. Figure 3 shows a drawing of the test rig used in production of the highspeed videos. The test section of the test rig has a window in which the high-speed camera can film the wave front at up 500000 frames per second.

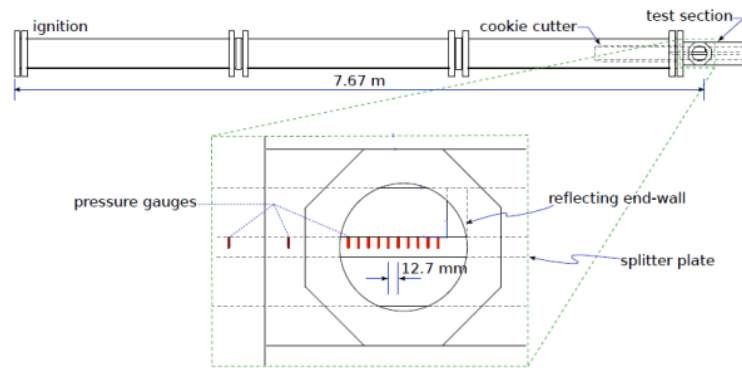

Figure 3. Drawing of the test rig used to produce shock wave experiments. Red lines in the splitter plate represents pressure sensors.

During the test procedure, the wave front hits the endwall in the test section and reflects causing the desired shock phenomena to occur. The splitter plate is equipped with pressure sensors, and measurements from these sensors can be used to calculate both the normal and the oblique shock velocity.
The splitter plate contains pressure sensors which are placed in the holes in the splitter plate. The accuracy of which the holes have been drilled has an impact when calculating wave front velocities, as the wave front velocities are sometimes upwards of $400 \quad[\mathrm{~m} / \mathrm{s}]$, depending on the gas being studied. Unfortunately, the technique based on pressure sensors is limited to spares measurements (1D+time). Even though, having velocities calculated through pressure sensors in addition to image processing creates a way to compare results. More importantly, with the framework based on image processing, the entire front is revealed (2D+time).

The outline of this paper is as follows: in Section 2, an edge detection algorithm based on Template Matching is introduced. Thereafter, a modified version of Segmented Regression is utilized to merge information generated by the Template Matching. In Section 3 the results are present, and the paper is closed with Conclusions in Section 4.

\subsection{Removal of Outlier Frames}

It was found that every $10^{\text {th }}$ image (i.e. no.: $1,11,21,31$ etc.) in the high-speed video films were severely corrupted by noise and blur compared to the rest of the film, due to hardware failure. These frames were therefore removed and are not expected to have significant influence on the final results. The removed frames must, however, be considered when estimating velocities, as the number of frames dictates the elapsed time of a wave front over a given distance.

\subsection{Template Matching}

Template Matching is used to generate two wave front tracking datasets. One template is designed to track the oblique shock segment, and one template is designed to track the normal shock segment. Both these datasets are later (see Subsection 2.3) processed by a modified Segmented Regression technique, leading to an accurate location of the entire front.

\subsubsection{Normal Shock Template}

Figure 4 illustrates the process of normal shock Template Matching using two 5-by-5 pixel matrices. Each of the templates (a 5-by-5 matrix) is assigned a pre-allocated value representative for the shock wave.

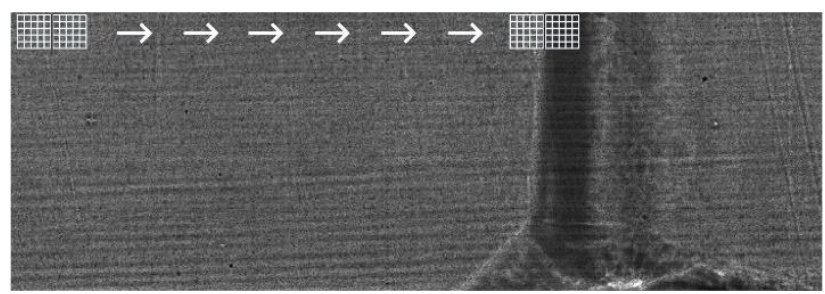

Figure 4. Normal shock Template Matching matrices on brightened raw image. Matrices are not to scale.

The left-hand template matrix is pre-allocated to best fit the brighter front portion of the wave front, while the 
right-hand template matrix is pre-allocated to best fit the darker portion within the wave front. The template is moved pixel by pixel, through the entire image. At each location, the deviation between the pre-allocated template value and all pixels covered by the template, is calculated. This error is then assigned to an error matrix, i.e. if the template is centered at row $i, \operatorname{column} j$, the deviation between the template and the pixels covered by the template, is assigned an error matrix at row $i$, column $j$. Results from the normal shock template matching is visualized in Figure 5.

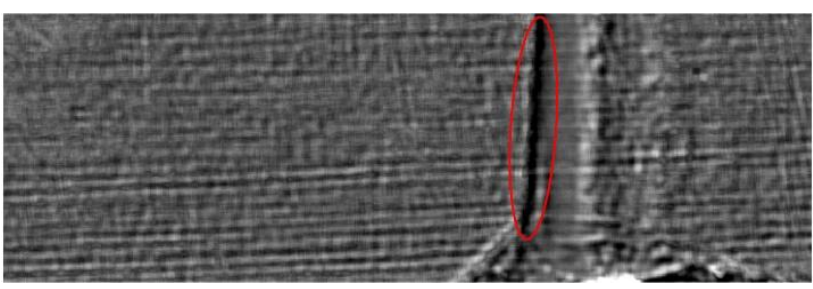

Figure 5. Error matrix produced by the normal shock Template Matching. A dark "ridge" is prominent where the normal shock is expected, indicated by the red ellipse.

The dataset for the normal shock is then generated from the error matrix by a simple approach; for each row, mark the position of the minimum value in the error matrix. The produced dataset is visualized as blue points in Figure 7. (a).

\subsubsection{Oblique Shock Template}

Figure 6 illustrates the process of oblique shock Template Matching using two 5-by-5 pixel matrices, both with the same pre-allocated average value, in a 45degree skewed orientation.

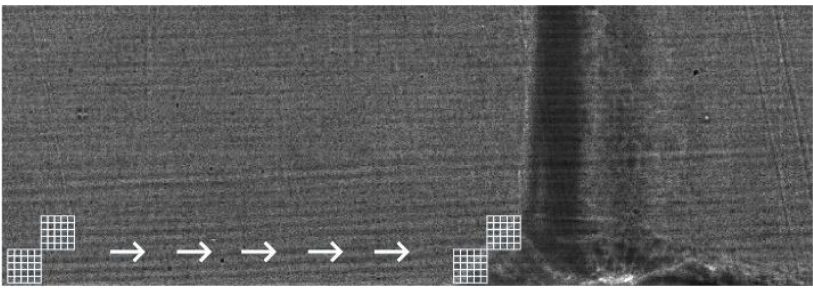

Figure 6. Oblique shock Template Matching matrices on brightened raw image. Matrices are not to scale.

The average value matches the brighter lower portion of the wave front, which is where the oblique shock occurs. At each location, the deviation between the preallocated template value and all pixels covered by the template, is calculated. This error is then assigned to an error matrix. The dataset for the oblique shock is then generated by the same approach as for the normal shock dataset, i.e. for each row, mark the position of the minimum value in the corresponding error matrix. The produced dataset is visualized as blue points in Figure 7. (b).

\subsection{Modified Segmented Regression}

A modified Segmented Regression technique is implemented to fit two line segments using two separate datasets. The modified approach is founded on the following observation; each of the templates is designed to track a specific feature in the shock wave. The normal shock template is designed to identify the vertical part of the shock wave, whereas the oblique shock template is designed to track the tilted part. However, the template designed to track normal shocks is, not expected to perform well in regions where oblique shocks occur, and vice versa. The datasets obtained through the Template Matching are illustrated in Figure 7. Please note that the normal shock template tracks the vertical part of the shock wave rather accurately, but fails to track the oblique part of the shock, see the lower part of Figure 7.(a). For the exact same input image, the oblique shock template tracks the tilted part of the shock wave rather accurately, but to some extent fails to track the vertical part of the shock, see the upper part of Figure 7.(b). Based on this, these two datasets should "merge" in such a way that coordinates describing the normal and the oblique shock, within these two datasets, are selected optimally.

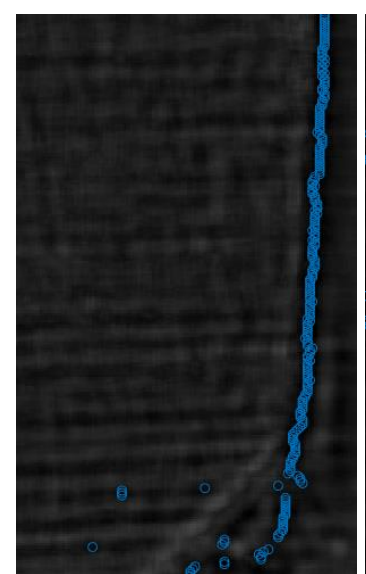

(a)

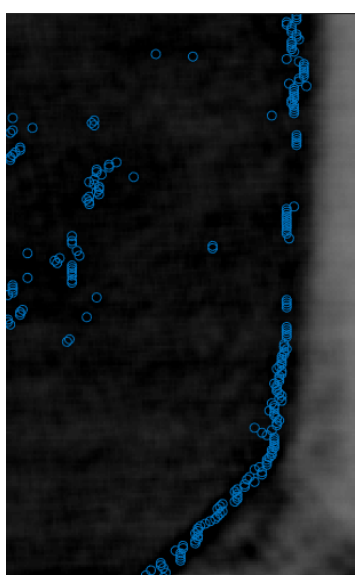

(b)
Figure 7. (a) and (b) are the resulting datasets of normal and oblique shock Template Matching.

To fit two line segments with the datasets illustrated in Figure 7, an optimization problem was formulated, and solved by a brute force approach:

$$
\begin{gathered}
S_{i}=\sum_{j=1}^{i}\left(\hat{y}_{j}-\left(a \hat{x}_{j}+b\right)\right)^{2} \\
+\sum_{j=i+1}^{n}\left(\bar{y}_{j}-\left(c \bar{x}_{j}+d\right)\right)^{2}, \\
\text { for } \mathrm{i}=2: \mathrm{n}-2
\end{gathered}
$$

where $S_{i}$ is a vector containing the combined error of both line segment estimates in each iteration, and $n$ is the number of rows in the image. Moreover, $\hat{y}_{j}, \hat{x}_{j}$ and 
$\bar{y}_{j}, \bar{x}_{j}$ represent the data points given by the normal and the oblique shock template, respectively. The constants $a, b$ and $c, d$ are the line segment coefficients representing the normal and oblique shock.

By minimizing $S_{i}$, the optimal combination of the two datasets is given. The index $i$ which corresponds to the lowest error $S_{i}$ becomes the breaking point. Meaning that coordinates situated at, and above, row number $i$ should be collected from the normal shock dataset, and coordinates situated below row number $i$ should be chosen from the oblique shock dataset. The result from the modified Segmented Regression technique is shown in Figure 8 .

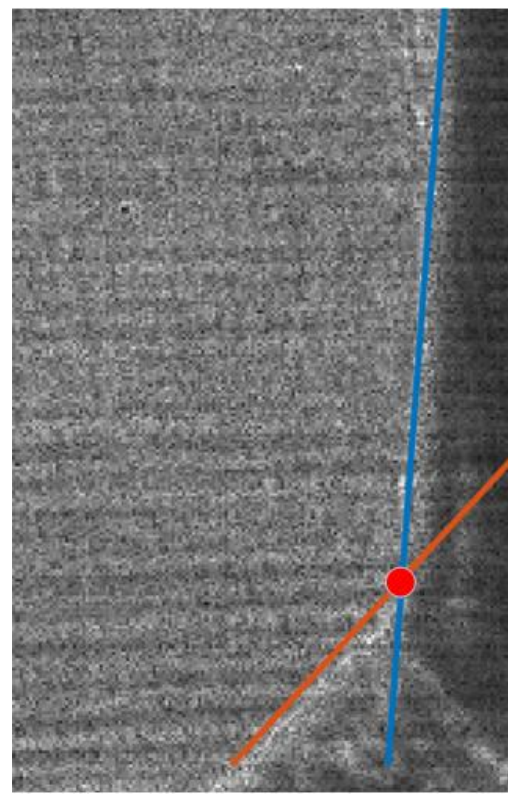

Figure 8. Resulting line segments obtained with a modified Segmented Regression approach. The red dot represents the breaking point, while the blue and orange line represents normal and oblique shock estimates, respectively.

\subsection{Normal and Oblique Shock Angle Estimation}

As first order polynomials are used to estimate both the normal and oblique shock, i.e. polynomials on the form $y=a x+b$, the slope of each shock is given by coefficient $a$, and thereby the angle is calculated by

$$
\alpha=\frac{\pi}{2}-\tan ^{-1}(a)
$$

Where $\alpha$ is the calculated angle and $a$ is the slope of the line segment representing the normal or the oblique shock. Formula (2) is applied to all wave front estimations throughout the videos, and the median of all normal angles is defined as $\alpha_{1}$ and the median of all oblique angles is defined as $\alpha_{2}$.

\subsection{Normal and Oblique Shock Velocity Estimation}

The velocity is estimated by comparing the position of the wave front in the first and the last image, relative to the time interval between the frames, i. e.

$$
v=\frac{\left(\Delta \mathrm{x} \cdot \mathrm{P}_{\frac{\mathrm{mm}}{\mathrm{px}}}\right)}{T_{\text {cam }} \cdot N_{\text {frames }}}
$$

where $v$ is the velocity of the shock wave in $\left[\frac{\mathrm{mm}}{\mathrm{s}}\right], \Delta \mathrm{x}$ is the number of pixels the shock wave has moved, $\mathrm{Pmm}_{\mathrm{px}}$ is a pixel to millimeter conversion factor, $T_{\text {cam }}$ is the elapsed time per frame capture and $N_{\text {frames }}$ is the number of frames the wave front is tracked.

The reason for calculating wave front velocities globally (first to last frame) instead of locally (two subsequent frames), is due to variations in the wave front estimate. Through visual inspection it comes clear that each wave front estimation might vary around $+/-7$ pixels while still being considered a satisfactory wave front estimate. In Figure 9, the span of an accepted wave front estimate is shown.

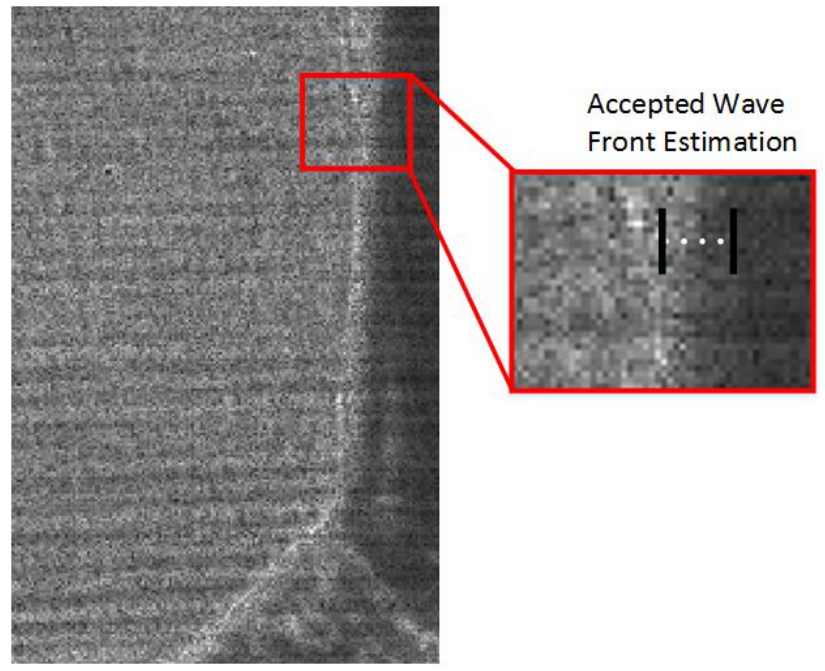

Figure 9. Illustration of expected wave front estimate. The black lines in the right-hand image displays the variation to be expected by a wave front estimate. The variation is around $+/-7$ pixels.

If the velocity is to be calculated from one frame to the next, $+/-7$ pixels could make a significant impact on the velocity estimate as a wave front moves approximately 5 pixels per frame. However, calculating the velocity over 100 frames, using the first and last frame, reduces the impact of wave front estimate variance.

A different solution to estimating the velocity could be calculating the velocity from one frame to the next for all frames, then find the median velocity of all the estimated velocities.

\section{Results}

The presented framework is tested on different highspeed videos to study the robustness of the framework. The results obtained through applying the framework to different high-speed videos are shown in Table 1 and Table 2 . 
Table 1. Velocity estimations of the shocks using the proposed framework.

\begin{tabular}{|l|l|l|l|}
\hline $\begin{array}{l}\text { Experime } \\
\text { nt no. }\end{array}$ & $\begin{array}{l}\text { Caption } \\
\text { freq. }\end{array}$ & $\begin{array}{l}\text { Normal } \\
\text { shock } \\
\text { velocity }\end{array}$ & $\begin{array}{l}\text { Oblique } \\
\text { shock } \\
\text { velocity }\end{array}$ \\
\hline 2516 & 500 & 230 & 262 \\
\hline 2533 & 200 & 471 & 473 \\
\hline 2543 & 200 & 236 & 268 \\
\hline 2573 & 500 & 220 & 261 \\
\hline 2592 & 500 & 450 & 486 \\
\hline
\end{tabular}

Table 2. Angle estimations of the shocks in the highspeed videos using proposed framework

\begin{tabular}{|c|c|c|}
\hline $\begin{array}{l}\text { Experime } \\
\text { nt no. }\end{array}$ & $\begin{array}{l}\text { Normal shock } \\
\text { angle }[\text { deg] }\end{array}$ & $\begin{array}{l}\text { Oblique shock } \\
\text { angle }[\text { deg }]\end{array}$ \\
\hline 2516 & 87 & 50 \\
\hline 2533 & 89 & 89 \\
\hline 2543 & 87 & 54 \\
\hline 2573 & 87 & 50 \\
\hline 2592 & 89 & 62 \\
\hline
\end{tabular}

Table 3 shows the confidence interval of the estimated normal and oblique shock angles.

Table 3. Confidence interval of normal and oblique shock angle estimations.

\begin{tabular}{|l|l|l|}
\hline $\begin{array}{l}\text { Experime } \\
\text { nt no. }\end{array}$ & $\begin{array}{l}\text { Normal shock } \\
\text { 95\% confidence } \\
\text { interval [deg] }\end{array}$ & $\begin{array}{l}\text { Oblique shock } \\
\text { 95\% confidence } \\
\text { interval }[\mathrm{deg}]\end{array}$ \\
\hline 2516 & {$[86 \ldots 87]$} & {$[47 \ldots 53]$} \\
\hline 2533 & {$[88 \ldots 91]$} & {$[88 \ldots 91]$} \\
\hline 2543 & {$[86 \ldots 88]$} & {$[50 \ldots 58]$} \\
\hline 2573 & {$[85 \ldots 87]$} & {$[45 \ldots 52]$} \\
\hline 2592 & {$[85 \ldots 93]$} & {$[57 \ldots 63]$} \\
\hline
\end{tabular}

A plot of all wave fronts tracked in experiment 2516 and 2573 can be seen in Figure 10 and Figure 11, respectively.

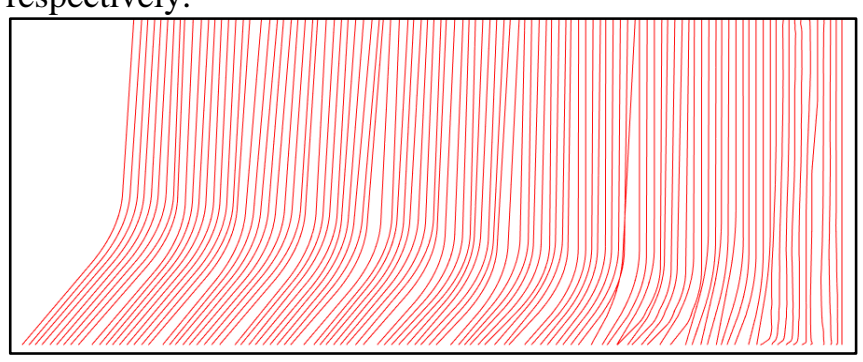

Figure 10. Result of all wave front estimations for experiment 2516 is displayed as red lines. The wave front in the first frame is the rightmost red line, while the wave front in the last frame is the leftmost red line.

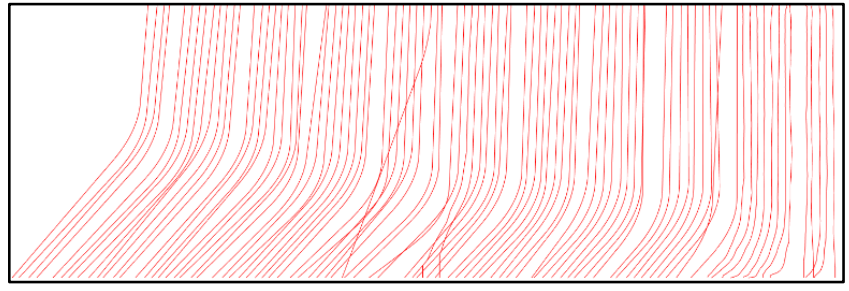

Figure 11. Result of all wave front estimations for experiment 2573 is displayed as the red lines. The wave front in the first frame is the rightmost red line, while the wave front in the last frame is the leftmost red line.

\section{Conclusions}

The task of developing a wave front tracking framework given by the Combustion, Process Safety and Explosions research group (CPSE) at University College of South-East Norway (USN) was approached with focus on finding a viable solution. As the highspeed videos provided is blurred and corrupted with noise, developing a solution which shows promise was prioritized. The result is a framework that estimates normal and oblique shock angle and velocity, as well as triple point estimation in each frame and triple point velocity in the given video. The framework is limited by the quality of the video provided, as the video is the only source of information the framework receives. Certain inputs from the user is required in order to ensure optimal performance of the framework.

The framework developed was first implemented on a single high-quality frame as a way to do a feasibility test during development. Secondly, the framework was expanded to automatically run for an entire video. Lastly, the framework was tested on different highspeed videos to test diversity of the framework.

An extended Segmented Regression method was developed in order to handle two separate data sets in parallel.

The proposed framework delivers results in accordance with theoretically calculated values and measured data presented by the CPSE research group at USN in cooperation with Caltech.

\section{References}

Adaptive-Vision (2017). "Template Matching". (Accessed 13.02.2017) URL: http://docs.adaptive-

vision.com/4.7/studio/machine_vision_guide/TemplateMat ching.html

R. Akbar (1997), "Mach Reflection of Gaseous Detonations". (Accessed 15.06.2017) URL: http://shepherd.caltech.edu/EDL/publications/reprints/akba rr_thesis.pdf

S. Bhardwaj, A. Mittal (2012). “A Survey on Various Edge Detector Techniques". (Accessed 10.03.2017)

URL: http://ac.els-cdn.com/S221201731200312X/1-s2.0S221201731200312X-main.pdf? tid=2e66faee-311c11e7-9ff2- 
00000aab0f01\&acdnat $=1493938456$ f3c2fa0b010f920c87 f9a17a81b5252e

A. Coste (2012). "Filtering, Edge detection and Template matching “. (Accessed 15.02.2017) URL:

http://www.sci.utah.edu/ acoste/uou/Image/project2/Arthu rCOSTE_Project2.pdf

N. Otsu, "A Threshold Selection Method from Gray-Level Histograms", IEEE Transactions on Systems, Man, and Cybernetics, Vol. 9, No. 1, 1979, pp. 62-66.

B. Schmidt (2013), "GALCIT 6-Inch Shock Tube". (Accessed 05.03.2017)

URL: http://shepherd.caltech.edu/T5/Ae104/Ae104b_hand out2013.pdf

B. E. Schmidt, B. Bobbitt, N. J. Parziale, and J. E. Shepherd (2013). "Experiments in a Combustion-Driven Shock Tube with an Area Change". (Accessed 19.05.2017) URL:

http://shepherd.caltech.edu/EDL/publications/reprints/024 6-000044.pdf

Wikipedia (January 2017), "Segmented Regression".

(Accessed 12.04.2017) URL:

https://en.wikipedia.org/wiki/Segmented_regression 